\title{
The belief-bias effect in the production and evaluation of logical conclusions
}

\author{
HENRY MARKOVITS and GUILAINE NANTEL \\ Université du Québec à Montréal, Canada
}

\begin{abstract}
In this study, we examined whether adult subjects' beliefs regarding the empirical truth of a conclusion affected their production as well as their evaluation of a logical conclusion in a reasoning task. In addition, the relation between the ability to resolve an abstract reasoning problem correctly and the effect of belief-bias was examined. The subjects were given one of four paper-andpencil reasoning tasks, two of them using an evaluation paradigm, and two of them using a production paradigm. Each paradigm comprised either neutral problems or belief problems. The neutral problems were constructed to be as similar as possible to the belief problems, in order to control for extraneous factors. All four tasks also included an abstract reasoning problem. The results indicate a significant belief-bias effect for both the evaluation and the production tasks. Qualitative analysis indicated that the belief-bias effect was more pervasive in the production condition. In addition, the belief-bias effect was found to exist independently of the subjects' abstract reasoning ability. The results are discussed with reference to a two-stage model, in which belief is used to resolve uncertainties in inferentially produced conclusions.
\end{abstract}

One of the more interesting phenomena in the research on reasoning concerns the belief-bias effect. Several researchers have claimed that subjects tend to evaluate the logical validity of deductive arguments on the basis of their personal beliefs regarding the empirical status of the conclusion. Specifically, subjects will tend to rate an argument as valid if they think that the conclusion is empirically true, and vice versa, irrespective of the textbook validity of the argument. The reality of the belief-bias effect has been questioned (Revlin \& Leirer, 1978; Revlin, Leirer, Yopp, \& Yopp, 1980), mainly on the grounds that some of the effects observed may be attributable to conversion effects due to subjects' idiosyncratic encoding of premises. However, Evans, Barston, and Pollard (1983) have demonstrated a strong belief-bias effect in experiments designed to control for both conversion and atmosphere effects.

The belief-bias effect has generally been found in paradigms for which subjects are presented with a specific conclusion (or a set of possible conclusions). It may be argued that, despite the most rigorous instructions, many subjects may be evaluating the empirical truth of the presented conclusion or conclusions and simply ignoring the premises, not because they cannot reason properly but because they do not properly understand the task (Henle, 1962). Such a possibility is certainly consistent with Evans

Preparation of this manuscript was supported by grants from the Quebec Ministry of Education (FCAR) and the Natural Sciences and Engineering Research Council of Canada. The authors would like to thank Robert Vachon for help in preparing and passing the questionnaires. They would also like to acknowledge the helpful comments of the anonymous reviewers of a previous version of the manuscript. Correspondence may be addressed to Henry Markovits, Département de psychologie, Université du Québec à Montréal, C.P. 8888, Succ. 'A'. Montréal, Québec H3C 3P8, Canada. et al.'s (1983) finding that subjects who accepted valid conclusions despite the influence of their beliefs referred more often to the premises than subjects who decided according to belief. One way of minimizing such a possibility and ensuring that subjects attend to the premises is to have subjects produce a conclusion. In other words, it may be argued that if the observed effects in evaluation tasks are due mainly to some process whereby subjects (mistakenly) examine only the conclusion of an argument, then forcing them to generate a conclusion from given premises should enable them to use their logical abilities more fully. Now, the effect of belief on the production of conclusions has been examined by Oakhill and Johnson-Laird (1985). They found that beliefs do tend to influence subjects' conclusions in a production task. However, they did not attempt to compare this effect with that found in an evaluation task. In addition, they compared subjects' performance with respect to believable as opposed to unbelievable conclusions, thus making the estimation of the magnitude of the effect somewhat difficult. The main aim of the present study was therefore to replicate the effects of subjects' beliefs on the production of conclusions in a reasoning task, and to compare this effect with that found in a comparable evaluation task.

In the present study, we also attempted to address two further questions. First, studies in conditional reasoning have clearly shown that subjects' performance is often influenced by a variety of factors that are not directly related to the logic of the premises or the conclusion, and that it varies according to the specific logical form employed or the content of the premises. Conversion effects (Revlin et al., 1980), atmosphere bias (Woodward \& Sells, 1935), figural effects (Johnson-Laird \& Steedman, 1978), and various forms of content effects (Guyotte \& Sternberg, 1981; O'Brien, Costa, \& Overton, 1986) are all ex- 
amples of such factors. Their existence makes comparisons among differing logical forms and contents somewhat hazardous. Note that Evans et al. (1983) explicitly controlled for conversion effects (as did Oakhill \& Johnson-Laird, 1985), atmosphere bias, and the figural effect. While there is no evidence to suggest that their results might nonetheless have been affected by some such (as yet unknown) factor, we felt that it would be useful to replicate the basic belief-bias effect by using a method that attempted to control explicitly for both form and content effects as tightly as possible. The method chosen involved the construction of pairs of categorical syllogisms. Each syllogism involved an initial premise (major term) of the form "all A are B," which was identical for both. The second premise took one of the following forms: (1) "X is $A$," (2) "X is $B$," (3) "X is not A," and (4) "X is not B." In all cases, $X$ was varied to produce two differing syllogisms: a neutral form that produced a conclusion for which the subjects had no a priori beliefs with respect to its empirical truth; and a positive form that produced a conclusion for which the subjects did have a clear belief regarding its empirical truth, and for which this belief contradicted the conclusion's logical status (i.e., if the subjects thought the conclusion was true, it was logically invalid, etc.). By comparing the subjects' performance on the neutral and positive forms, it was felt that the belief-bias effect could be examined in a way that would eliminate any possible effects related to logical form and would minimize content variations.

The final question addressed by this study concerns the possible relation between logical competence and beliefbias. Evans et al. (1983) found evidence that subjects may oscillate between competing logical and nonlogical processes. This implies that subjects do attempt to consider the logical validity of an argument by examining the premises, when they are not led to employ other criteria such as their beliefs. Without entering into the problem associated with the nature of logical competence, it appears reasonable to suppose that if subjects have difficulty in reasoning correctly from premises that are relatively content-free or abstract, they might be more prone to use nonlogical indices when these are present. Thus there should be a relation between the ability to reason correctly during a content-free task and the performance on a task for which a belief-bias effect may exist.

\section{METHOD}

\section{Materials}

Four paper-and-pencil questionnaires were constructed. The first page of each questionnaire, which was identical for each one, presented a series of four conditional reasoning problems with abstract content. At the top of the page was written:

Suppose it is true that:

All the XAR's are YOF's

and answer the multiple choice questions.

After this came four multiple-choice questions. The first one took the following format:
(A) If a glock is a XAR, you can say

(a) that it is certain that the glock is a YOF.

(b) that it is certain that the glock is not a YOF.

(c) that it is not certain whether the glock is a YOF or not.

The three other questions used the same format and presented the following statements: "(B) If a koy is a YOF, you can say"; "(C) If a glock is not a XAR, you can say"; "(D) If a koy is not a YOF, you can say." These four correspond to the logical forms modus ponens, converse, inverse, and contrapositive.

Note that in all cases, the abstract content questions preceded the syllogisms that were to examine the belief-bias effect. It has been shown that such a procedure encourages a logical reasoning mode in subjects that diminishes content effects (Hawkins et al., 1984; Markovits, in press). This procedure was thus designed to reduce any possible belief-bias effect and to facilitate the effect of subsequent instructions.

Two questionnaires used an evaluation paradigm. For these, the second page contained the following instructions (adapted from Evans et al., 1983):

You are going to receive a series of eight problems. You must decide whether the stated conclusion follows logically from the premises or not.

You must suppose that the premises are all true and limit yourself only to the information contained in these premises. This is very important.

At the top of the next page, the subjects received the following instructions (adapted from Evans et al., 1983):

For each problem, decide if the given conclusion follows logically from the premises. Circle YES if, and only if, you judge that the conclusion can be derived unequivocally from the given premises, otherwise circle NO.

For the positive evaluation questionnaire, subjects were then presented with the following eight syllogisms (four to a page):

(1) Premise 1: All things that are smoked are good for the health. Premise 2: Cigarettes are smoked.

Conclusion: Cigarettes are good for the health.

(2) Premise 1: All unemployed people are poor.

Premise 2: Rockefeller is not unemployed.

Conclusion: Rockefeller is not poor.

(3) Premise 1: All flowers have petals. Premise 2: Roses have petals. Conclusion: Roses are flowers.

(4) Premise 1: All animals with four legs are dangerous. Premise 2: Poodles are not dangerous. Conclusion: Poodles do not have four legs.

(5) Premise 1: All mammals walk. Premise 2: Whales are mammals. Conclusion: Whales walk.

(6) Premise 1: All eastern countries are communist. Premise 2: Canada is not an eastern country. Conclusion: Canada is not communist.

(7) Premise 1: All animals love water. Premise 2: Cats do not like water Conclusion: Cats are not animals.

(8) Premise 1: All things that have a motor need oil. Premise 2: Automobiles need oil. Conclusion: Automobiles have motors.

These syllogisms include two $(1,5)$ of the form "all A are B, C are $A$, thus $C$ are $B, "$ and two $(4,7)$ of the form "all $A$ are $B$, $C$ are not $B$, thus $C$ are not $A$." In each of them, the conclusions 
were rated as unbelievable by 37 independent subjects (see Table 1), and, in each case, the conclusion was logically valid. In addition, there are two syllogisms $(3,8)$ of the form "all A are $B, C$ are $B$, thus $C$ are $A$," and two $(2,6)$ of the form "all $A$ are $B, C$ are not A, thus $\mathbf{C}$ are not B." In each of these cases, the conclusions were rated as believable and were logically invalid. In the latter two cases, it should be noted that the forms are indeterminatethat is, there is no logically valid conclusion.

The second questionnaire that used the evaluation paradigm (neutral evaluation) was identical to the first, but with one major difference. In all cases, the minor premise of the eight syllogisms was altered in order to make the conclusion neutral with respect to belief (see Table 1). The resulting conclusions were:

(1) Ramadions are good for the health.

(2) Hudon is not poor.

(3) Pennes are flowers.

(4) Argomelles do not have four legs.

(5) Lapitars walk.

(6) Sylvania is not communist.

(7) Selacians are not animals.

(8) Opprobines have motors.

In addition to the two questionnaires that used an evaluation paradigm, two more that used a production paradigm were constructed. The first page of these two questionnaires, as stated above, was identical to the one described previously: it contained a set of four conditional reasoning problems with abstract content. On the second page of both production questionnaires was written the following:

You are going to receive a series of eight problems. You must produce a conclusion which follows logically from the premises.

You must suppose that the premises are all true and limit yourself only to the information contained in these premises. This is very important.

At the top of the next page, the subjects received the following instructions:

For each problem, give the conclusion which follows logically from the premises. Formulate a conclusion only if you judge that it is possible to derive one unequivocally from the given premises. If you think that no conclusion can be logically and unequivocally derived from the premises, write NONE.

\section{Table 1}

Average Mean Believability Ratings and Standard Deviations for Pairs of Conclusions (Positive [P] and Neutral [N] forms) on a Scale from -3 (Certainly False) to +3 (Certainly True)

\begin{tabular}{clrr}
\hline Form & \multicolumn{1}{c}{ Conclusion } & Mean & $S D$ \\
\hline P & Cigarettes are good for the health & -2.73 & 1.02 \\
N & Ramadions are good for the health & -0.11 & .61 \\
P & Rockefeller is not poor & +2.27 & 1.68 \\
N & Hudon is not poor & +0.30 & .66 \\
P & Roses are flowers & +2.92 & .36 \\
N & Pennes are flowers & -0.43 & 1.09 \\
P & Poodles do not have four legs & -2.54 & 1.45 \\
N & Argomelles do not have four legs & +0.05 & .33 \\
P & Whales walk & -2.76 & 1.04 \\
N & Lapitars walk & +0.35 & .82 \\
P & Canada is not communist & +2.32 & 1.31 \\
N & Sylvania is not communist & -0.03 & 1.14 \\
P & Cats are not animals & -2.84 & .99 \\
N & Selaciens are not animals & +0.05 & 1.05 \\
P & Automobiles have motors & +2.84 & .83 \\
N & Opprobines have motors & -0.19 & .78 \\
\hline
\end{tabular}

After this, one of the two series of eight syllogisms that were used in the evaluation questionnaires was presented (leading to two forms, neutral production and positive production), with the difference that no conclusion was presented. Space was provided for the subjects to write down a conclusion.

\section{Procedure}

The four forms of questionnaire were distributed at random among entire classes of university students. The subjects were informed that there was no time limit and that they could proceed until satisfied with their answers.

\section{Subjects}

A total of 186 French-speaking university students received one of four questionnaires. Of these, 44 received the positive production form; 48 , the neutral production form; 43 , the positive evaluation form; and 51 , the neutral evaluation form.

\section{Scoring}

The subjects were scored both on the abstract conditional reasoning problem and on the eight syllogisms with content. For the abstract problems, the subjects' responses were rated conditional (for those who responded correctly to all four forms), intermediate (correct response to modus ponens, incorrect response to one or more of the three other forms), or biconditional (responding to all four forms as if the relation were "if and only if"). In addition, two response patterns were taken to indicate difficulty in accepting the given premises (Markovits, in press). Thus those subjects who gave a response of uncertainty to modus ponens, or who responded by inverting the truth value of the minor premise in the conclusion (e.g., "If $P$ then $Q, Q$ is true, then $P$ is false"), were rated as having given an uncertain response to the abstract conditionals. In the present context, the rationale for such a scoring schema derives from the following considerations: The logically correct response to the four conditional forms is the conditional. Previous studies have indicated that the probability that a subject will produce the logically correct response to subsequent problems varies with the response that the subject gives on an initial problem (Markovits, 1984, in press), going from conditional, to intermediate, to biconditional, to uncertain. Thus, the scoring schema employed here explicitly uses the probability of producing the logically correct response to conditional syllogisms as an indication of reasoning ability.

Scoring on the positive evaluation syllogisms was as follows. The subjects were given one point for each time they decided that a believable response was valid or an unbelievable response was invalid. The scores thus ranged from 0 to 8 . For the neutral evaluation syllogisms, the subjects were given a score of 1 for each time they gave a response equivalent to that which was scored on the positive form. Thus a subject who decided that "cigarettes are good for the health" was invalid on the positive form received 1 point, while a subject who decided that "ramadions are good for the health" was invalid on the neutral form also received 1 point. The score on the neutral evaluation syllogisms thus indicates the number of times that the subjects chose the believable response for reasons related to either the logical form or the global content of the syllogisms used here, not to the believability of the conclusion.

Scoring on the positive production syllogisms was as followsfor the sake of brevity, in the following, the logical form will be referred to by the minor premise only (i.e., "A is B and C is A" will be designated by " $C$ is $A$ "): For the two indeterminate forms (" $C$ is $B, "$ " $C$ is not $A$ "), the subjects were given 1 point each time they produced a logically invalid but believable conclusion. For example, for the syllogism " all flowers have petals; roses have petals," the subjects who gave the conclusion "roses are flowers", would receive 1 point. For the other two forms ("C is $A$," "C is not B"), the subjects received 1 point each time they did not 
produce the logically valid but unbelievable conclusion. For example, for the syllogism "all things that are smoked are good for the health; cigarettes are smoked," the subjects received 1 point if they concluded that "cigarettes are not good for the health," or if they did not give a firm conclusion (e.g., "cigarettes may or may not be good for the health"). For the neutral production syllogisms, the subjects were given 1 point each time they produced (or did not produce) responses equivalent to those for the positive syllogisms. These responses were identical to those in the positive form, with exception for the substituted term. For the two indeterminate syllogisms (" $\mathrm{C}$ is $\mathrm{B}$," " $\mathrm{C}$ is not $\mathrm{A}$ "), this implied that responses such as "pennes are flowers" received 1 point. For the two other forms ("C is A," "C is not B"), subjects responding with, for example, "ramadions are not good for the health" or subjects not giving a firm conclusion received 1 point. Note that the scores on the production syllogisms are directly comparable to the scores on the evaluation forms, if one assumes that a subject who produces a given conclusion would evaluate that conclusion as valid and would evaluate a different conclusion as invalid. For ease of reference, these scores will be referred to as belief scores.

\section{RESULTS}

Table 2 indicates the average belief scores for each of the four logical forms (two problems per form) as a function of type of syllogism and form of presentation. An analysis of variance using belief scores as the response variable, and type of syllogism (neutral or positive), form of presentation (evaluation or production), and performance on the abstract problems (conditional, intermediate, biconditional or uncertain) as factors was performed. Following Conover (1980), this analysis was repeated after converting the scores on the syllogisms to rank orders. Since both analyses gave substantially the same results, only the first will be reported. This indicated significant main effects for type of syllogism $[F(1,170)=20.39, M S$ e $=62.2, p<.01]$, form of presentation $[F(1,170)=$ $3.94, M S \mathrm{e}=12.03, p<.05$ ], and performance on the abstract problem $[F(3,170)=9.64, M S e=23.4$, $p<.01]$. None of the interaction terms was significant. The first two main effects indicate that belief scores were higher for positive than for neutral forms, and higher for production than for evaluation. The third main effect is due to scores' generally being lower for subjects giving conditional responses to the abstract problem (mean score $=1.92$ ) than for those giving intermediate responses (mean $=2.64)$, which were in turn lower than for subjects giving biconditional (mean $=3.63$ ) or uncertain responses (mean $=3.74$ ).

These results clearly support the general thesis of the existence of a belief-bias effect in reasoning. However, the idea that the production condition should generate a lower level of belief-bias is not supported. In accord with the results obtained by Marcus and Rips (1979), the production condition produced generally higher scores than the evaluation condition, although the effect of belief was present for both conditions. In this context, it is interesting to take a more qualitative look at the results. Four logical forms were examined with the eight syllogisms, two of each. Table 2 indicates the average belief score (or the equivalent in the neutral forms) for the two cases, for each of the four forms. An initial question concerns the generality of the belief-bias effect across specific contents. The Mann-Whitney procedure was used to examine the effect of belief-bias for single items. This indicated that for the evaluation condition, a significant effect was found for two of the eight items. For the production condition, a significant effect was found for five of eight items, and a sixth item was significant at the .06 level. The two items for which no significant difference was found for the production condition were those employing modus ponens ( $P$ is true), for which almost no belief-bias effect was obtained for either condition. Thus, the overall belief-bias effect is generalized across the various contents employed here.

Inspection of Table 2 shows that for the evaluation condition, the major effect of belief-bias is concentrated on the two indeterminate forms (" $\mathrm{C}$ is not $\mathrm{A}$," " $\mathrm{C}$ is $\mathrm{B}$ "). Mann-Whitney procedures indicate that for each of these, the scores are significantly higher for the positive than for the neutral forms $(z=1.86, p<.05 ; z=1.98$, $p<.05$ ). This is consistent with previous results (Evans et al., 1983; Oakhill \& Johnson-Laird, 1985). Note that while many subjects had difficulty reasoning with the " $\mathrm{C}$ is not B" form (as indicated by the relatively high belief score on the neutral form), the effect of belief was quite small $(z=0.61$, n.s. $)$. In the production condition, the effect of belief is also significant for the indeterminate forms, " $\mathrm{C}$ is not $\mathrm{A}$ " $(z=2.31, p<.02)$ and " $\mathrm{C}$ is $\mathrm{B}$ "

Table 2

Average Belief Scores (Maximum Score of 2) for the Four Logical Types as a Function of Mode of Presentation (Evaluation or Production) and Content (Neutral or Positive)

\begin{tabular}{lccccc}
\hline & \multicolumn{2}{c}{ Evaluation } & & \multicolumn{2}{c}{ Production } \\
\cline { 2 - 3 } \cline { 5 - 6 } Logical Form & Neutral & Positive & & Neutral & Positive \\
\hline A is B, C is A & 0.20 & 0.26 & & 0.33 & 0.43 \\
$\mathrm{~A}$ is B, C is B & 0.86 & 1.23 & & 0.79 & 1.48 \\
$\mathrm{~A}$ is B, C is not A & 0.59 & 0.93 & 0.58 & 0.98 \\
$\mathrm{~A}$ is B, C is not B & 0.71 & 0.63 & & 0.69 & 1.00 \\
Total (maximum = 8) & 2.36 & 3.05 & & 2.39 & 3.89 \\
\hline
\end{tabular}


$(z=3.90, p<.001)$. In this case, however, there is a marginally significant difference for the " $\mathrm{C}$ is not $\mathrm{B}$ " form $(z=1.64, p<.055)$.

In addition to verifying the existence of belief-bias effects, the method employed here permits some evaluation of the extent to which belief-bias exerts an influence on reasoning independently from any other variable. Table 2 indicates that the subjects examined here produced an overall error rate of close to $30 \%$ in reasoning with syllogisms for which possible conclusions were neither believable nor unbelievable (neutral forms), in both the evaluation and production conditions. A direct comparison of overall error rates indicates that the presence of belief-bias produced an increase of $30 \%$ in the error rate in the evaluation condition, and a corresponding increase of $63 \%$ in the production condition. This suggests that while the presence of belief-bias does significantly influence adult reasoning, it is clearly the case that beliefbias alone cannot account for the majority of the errors that subjects make on items for which belief-bias may be present. These overall results must be qualified as a function of the specific logical form examined. If one considers the two indeterminate forms for which the effect of belief was significant for both the evaluation and production conditions, the relative increases in the error rate due to belief-bias were $49 \%$ and $80 \%$, respectively. For the two other forms, "C is A" and " $\mathrm{C}$ is not $\mathrm{B}$," the relative increases were $2 \%$ and $40 \%$, respectively.

In addition, these results, coupled with previous data concerning differences for individual items, suggest that belief-bias appears to be more pervasive in the production task than in the evaluation task. In the latter case, the effects of belief are more limited, both in overall scope and in the number of specific items significantly affected.

Now, in the evaluation condition, the judgment that a conclusion is invalid could imply that the subject feels that there is no valid conclusion, or that the subject feels that a conclusion other than the one presented is valid. In the production condition, subjects must produce a specific conclusion, and this condition thus permits a more specific coding of the results than does the evaluation condition. Table 3 presents the proportion of the three possible responses observed for each of the four logical forms. Inspection of this table suggests two interesting effects. First, for the two determinate forms (" $\mathrm{C}$ is $\mathrm{A}$," " $\mathrm{C}$ is not $B$ "), the effect of belief results in a higher proportion of no specific conclusion, not in any tendency to produce the overtly believable response. This is consistent with results obtained by Oakhill and Johnson-Laird (1985). For example, for the syllogism, "all eastern countries are communist; Canada is not an eastern country," subjects tend to give no firm conclusion rather than to produce the believable conclusion that "Canada is not communist." Second, there appears to be at least a rough relation between problem difficulty as measured by the proportion of logically correct responses on the neutral form and the effect of belief. The " $\mathrm{C}$ is $\mathrm{A}$ " form has the highest proportion of logically correct responses and the " $\mathrm{C}$ is B" has the lowest such proportion. These forms show, respectively, the least and greatest effect of belief.

Finally, the relation between the belief-bias effect and reasoning ability merits a more detailed examination. Table 4 indicates the average scores on the eight syllogisms across both the evaluation and production conditions for subjects giving conditional, intermediate, biconditional, or uncertain responses to the abstract problems. Inspection of this table shows that belief scores for positive content increase as reasoning performance on the abstract task decreases. However, part of this increase is attributable to the fact that less able subjects tend more often to fall into reasoning errors when belief does not play a part. Although the difference between belief scores for neutral and those for positive content does increase somewhat with decreasing performance on the abstract problem, this effect is not significant.

\section{DISCUSSION}

The results of the present study add to the weight of the evidence that indicates a significant effect of subjects'

Table 3

Proportion of Observed Responses for the Four Logical Forms in the Production Condition for Neutral and Positive Content

\begin{tabular}{|c|c|c|c|}
\hline \multirow[b]{2}{*}{ Logical Form } & \multirow[b]{2}{*}{ Response } & \multicolumn{2}{|c|}{ Content } \\
\hline & & Neutral & Positive \\
\hline$A$ is $B, C$ is $A$ & $\begin{array}{l}{ }^{*} \mathrm{C} \text { is } \mathrm{B} \\
+\mathrm{C} \text { is not } \mathrm{B} \\
\text { tno specific conclusion }\end{array}$ & $\begin{array}{l}.84 \\
.00 \\
.16\end{array}$ & $\begin{array}{l}.76 \\
.03 \\
.21\end{array}$ \\
\hline$A$ is $B, C$ is $B$ & $\begin{array}{l}+C \text { is } A \\
C \text { is not } A \\
*_{\text {no specific conclusion }}\end{array}$ & $\begin{array}{l}.40 \\
.01 \\
.59\end{array}$ & $\begin{array}{l}.74 \\
.00 \\
.26\end{array}$ \\
\hline$A$ is $B, C$ is not $A$ & $\begin{array}{l}\text { C is B } \\
\dagger C \text { is not B } \\
\text { *no specific conclusion }\end{array}$ & $\begin{array}{l}.03 \\
.29 \\
.68\end{array}$ & $\begin{array}{l}.00 \\
.49 \\
.51\end{array}$ \\
\hline$A$ is $B, C$ is not $B$ & $\begin{array}{l}+C \text { is } A \\
{ }^{*} \mathrm{C} \text { is not } A \\
\text { tno specific conclusion }\end{array}$ & $\begin{array}{l}.01 \\
.67 \\
.32\end{array}$ & $\begin{array}{l}.02 \\
.52 \\
.46\end{array}$ \\
\hline
\end{tabular}

*The logically correct response. $\quad+$ Response(s) associated with belief-bias effect. 
Table 4

Average belief scores (Combining evaluation and Production Conditions) for Neutral and Positive Content as a Function of Subjects' Responses to the Abstract Problems

\begin{tabular}{ccc}
\hline \multirow{2}{*}{$\begin{array}{c}\text { Response to } \\
\text { Abstract Problem }\end{array}$} & \multicolumn{2}{c}{ Content } \\
\cline { 2 - 3 } & Neutral & Positive \\
\hline Conditional & 1.50 & 2.33 \\
Intermediate & 2.13 & 3.15 \\
Biconditional & 2.83 & 4.43 \\
Uncertain & 3.18 & 4.29 \\
\hline
\end{tabular}

beliefs on logical reasoning tasks. By comparing performance on the positive and neutral forms of the syllogisms used in the study, one may discount any explanation that might claim that subjects' choices on the positive forms are based on some (known or unknown) strategy that does not reflect their beliefs.

Our expectation that the belief-bias effect would be less evident if subjects were asked to produce their own conclusions than if asked to evaluate the validity of a presented conclusion was not confirmed. Instead, the results indicate that subjects' beliefs exert a more pervasive influence on production of their own conclusions than on their evaluations of presented conclusions. Now, it may well be the case that the processes by which belief-bias operates differ in the evaluation and in the production paradigm. For example, some subjects may tend simply to ignore the premises in the evaluation task, as suggested by Evans et al. (1983). Such a strategy is clearly not possible in the production task. In fact, the existence of the beliefbias effect in the production task has some implications that concern the nature of reasoning. In the production condition, subjects must rely on the premises to generate a conclusion. The existence of a belief-bias effect thus indicates that many subjects either employ highly contextspecific rules of combination or employ a verification process that reexamines any produced conclusion for validity.

Any attempted explanation of how the belief-bias effect operates in the production task must take into account the way subjects might be processing the premises. In turn, it must also take into account the differences observed in problem type. The major part of the belief-bias effect in the present study involved deducing an invalid, but believable, conclusion, in accord with previous results (Evans et al., 1983; Oakhill \& Johnson-Laird, 1985). Now, an invalid conclusion often has the property that it might be true in some cases, but not true always. Thus, for example, the premises "all flowers have petals; pennes have petals" are consistent with both the statement that "pennes are flowers"' and the statement that "pennes are not flowers." In fact, analysis of written responses to such syllogisms indicated a relatively high frequency of explicit responses of this kind (such as, "pennes may or may not be flowers," etc.). This suggests that the subjects were generally using a process of inference from premises to generate their conclusions. When a subject produces competing and incompatible responses through an initial process of inference, belief-bias effects are clearly present. In this case, it is possible that the subjects were using belief in order to choose between the two responses, that is, as a second-order strategy. It may thus be the case that subjects use both a logical strategy and a nonlogical strategy in the same context, with the latter being used in order to accommodate a degree of internal conflict or uncertainty.

This analysis is reinforced by the observation that on the forms " $\mathrm{C}$ is $\mathrm{A}$ " and " $\mathrm{C}$ is not $\mathrm{B}$," for which the $\log$ ically correct conclusion was unbelievable, relatively few subjects produced a believable conclusion. Instead, the effect of belief-bias translated into a greater proportion of uncertain conclusions. This is also consistent with the idea that subjects often produce a conclusion based on inferential reasoning, but that subsequent evaluation of this conclusion may result in the introduction of real-world knowledge.

These results thus suggest that subjects employ a twostage strategy in the production of logical conclusions. The first stage involves the generation of a conclusion (or conclusions) by a process of reasoning; the second involves a process of evaluation during which extralogical factors such as belief might enter in. The results also indicate that the weight of the second process might be mediated by the difficulty of the problem. Thus, the probability that subjects will reevaluate a conclusion might depend on the degree of certainty that they attribute to it.

Finally, the ability to reason given abstract content does appear to be related to subsequent reasoning given syllogisms with concrete content. However, it is not the case that subjects who reason better abstractly are impervious to belief-bias effects. Although there is some such effect, the results are consistent with an additive model, in which the effect of belief is superimposed upon a subject's ability to give the logically correct answer. This latter is also consistent with our results on the relative magnitude of belief-bias effects. Comparison between neutral and positive forms indicates that even on items for which beliefbias is present, a majority of errors in reasoning can be attributed to factors unrelated to subjects' beliefs. In other words, belief-bias appears to influence subjects at a stage beyond that at which they generate conclusions by normal inferential processes. Note that this is also consistent with the idea that belief-bias is present when subjects are not certain about the inferential status of a conclusion.

Overall, these results thus tend to confirm the reality of the belief-bias effect. They are not readily explainable by artifactual effects due to form or content of the syllogisms, nor by misunderstanding of the instructions as might be the case in the evaluation task. However, they are also compatible with an explanation that supposes that subjects do reason (more or less well) from the premises 
when faced with a logical problem, but may use belief at a subsequent stage in order to resolve an uncertain conclusion.

\section{REFERENCES}

Conover, W. J. (1980). Practical non-parametric statistics. New York: Wiley.

Evans, J. St. B. T., Barston, J. L., \& Pollard, P. (1983). On the conflict between logic and belief in syllogistic reasoning. Memory \& Cognition, 11, 295-306.

Guyotre, M. J., \& Sternberg, R. J. (1981). A transitive-chain theory of syllogistic reasoning. Cognitive Psychology, 13, 461-525.

Hawkins, J., Pea, R. D., Glick, J., \& Scribner, S. (1984). “Merds that laugh don't like mushrooms": Evidence for deductive reasoning by preschoolers. Developmental Psychology, 20, 584-594.

Henle, M. (1962). On the relation between logic and thinking. Psychological Review, 69, 366-378.

Johnson-Laird, P., STEedman, M. (1978). The psychology of syllogisms. Cognitive Psychology, 10, 64-99.

MarCuS, S. L., \& RIPS, L. J. (1979). Conditional reasoning. Journal of Verbal Learning \& Verbal Behavior, 18, 199-223.

Markovits, H. (1984). Awareness of the 'possible' as a mediator of formal thinking in conditional reasoning problems. British Journal of Psychology, 75, 367-376.

MARKovits, H. (in press). Reasoning with contrary-to-fact propositions. Journal of Experimental Child Psychology.

O'Brien, D. P., Costa, G., Overton, W. F. (1986). Evaluations of causal and conditional hypotheses. Quarterly Joumal of Experimental Psychology, 38A, 493-512.

OAKHILL, J. V., Johnson-LaIRD, P. N. (1985). The effects of belief on the spontaneous production of syllogistic conclusions. Quarterly Journal of Experimental Psychology, 37A, 553-569.

REvLIN, R., \& LeIRER, V. (1978). The effects of personal biases on syllogistic reasoning: Rational decisions from personalized representations. In R. Revlin \& R. E. Meyer (Eds.), Human reasoning (pp. 5182). Washington: Winston-Wiley.

Revlin, R., Leirer, V., YoPp, H., \& Yopp, R. (1980). The beliefbias effect in formal reasoning: The influence of knowledge on logic. Memory \& Cognition, 8, 584-592

Woodworth, R. S., \& SELLS, S. B. (1935). An atmosphere effect in syllogistic reasoning. Journal of Experimental Psychology, 18, $451-460$

(Manuscript received May 20, 1987; revision accepted for publication March 12, 1988.) 